\title{
Pacific
}

Journal of

Mathematics

LINEAR COMBINATIONS OF LOGARITHMIC DERIVATIVES OF ENTIRE FUNCTIONS WITH APPLICATIONS TO DIFFERENTIAL EQUATIONS

JOSEPH B. MiLes AND JOHN Rossi 


\section{LINEAR COMBINATIONS OF LOGARITHMIC DERIVATIVES OF ENTIRE FUNCTIONS WITH APPLICATIONS TO DIFFERENTIAL EQUATIONS}

\section{J. Miles AND J. Rossi}

Let $F_{1}, F_{2}, \ldots, F_{L}$ be entire functions of finite order and let $c_{1}, c_{2}, \ldots, c_{L}$ be complex numbers whose convex hull does not contain 0 . A lower bound in terms of the counting functions of the zeros of the $F_{j}^{\prime} s$ is obtained for

$$
\left|\sum_{j=1}^{L} c_{j} r e^{i \theta} F_{j}^{\prime}\left(r e^{i \theta}\right) / F_{j}\left(r e^{i \theta}\right)\right|
$$

valid for $r$ in a set of positive logarithmic density and $\theta$ in a set $U_{r} \subset[0,2 \pi]$ of fixed positive measure. This bound is used to extend a result of Bank and Langley concerning the exponent of convergence of the zero sequences of solutions of certain linear differential equations with entire coefficients.

\section{Introduction.}

In this paper we are concerned with the behavior of the logarithmic derivative $F^{\prime} / F$ of an entire function $F$ of finite order, and most particularly with lower bounds for $\left|F^{\prime} / F\right|$. From the argument principle it follows that if $F$ has no zeros on $|z|=r$, then

$$
\frac{1}{2 \pi} \int_{0}^{2 \pi} r e^{i \theta} \frac{F^{\prime}\left(r e^{i \theta}\right)}{F\left(r e^{i \theta}\right)} d \theta=n(r, 0, F)
$$

where $n(r, 0, F)$ denotes the number of zeros of $F$ in $|z| \leq r$ counting multiplicity. Our principal result implies for most values of $r$ that if $0<\beta<$ 1 , then the modulus of the above integrand is greater than or equal to $\beta n(r, 0, F)$ on a substantial portion of the circle $|z|=r$.

For applications to the behavior of solutions of certain differential equations it is useful to formulate our result in terms of linear combinations of logarithmic derivatives.

Theorem 1. Suppose $F_{j}, 1 \leq j \leq L$, are entire functions each with order not exceeding $\rho<\infty$. Suppose $c_{j}, 1 \leq j \leq L$, are complex numbers lying in a 
sector with vertex at the origin and angle opening $2 \gamma$ for some $\gamma$ in $[0, \pi / 2)$. For $\beta \in(0,1)$ and $r>0$, let

$$
U_{r}=\left\{\theta \epsilon[0,2 \pi]:\left|\sum_{j=1}^{L} c_{j} r e^{i \theta} \frac{F_{j}^{\prime}\left(r e^{i \theta}\right)}{F_{j}\left(r e^{i \theta}\right)}\right| \geq \beta\left|\sum_{j=1}^{L} c_{j} n\left(r, 0, F_{j}\right)\right|\right\} .
$$

Then for $M>3 L$ there exists $E=E_{M} \subset[1, \infty)$ with lower logarithmic density at least $1-3 L / M$ such that

$$
m\left(U_{r}\right)>\left(\frac{(1-\beta) \cos \gamma}{7 M(\rho+1)}\right)^{2}, \quad r \in E
$$

Here $m$ denotes Lebesgue measure. The upper and lower logarithmic density of $E \subset\left[R_{0}, \infty\right)$ for some $R_{0}>0$ are defined by

$$
\overline{\log \operatorname{dens}} E=\varlimsup_{r \rightarrow \infty} \frac{1}{\log r} \int_{E \cap\left[R_{0}, r\right]} \frac{d t}{t}
$$

and

$$
\underline{\log \operatorname{dens}} E=\underline{\lim }_{r \rightarrow \infty} \frac{1}{\log r} \int_{E \cap\left[R_{0}, r\right]} \frac{d t}{t}
$$

respectively.

We make several observations concerning Theorem 1. First, Theorem 1 asserts that $m\left(U_{r}\right)$ is bounded away from 0 for $r \in E$ whenever 0 does not lie in the convex hull of $\left\{c_{j}\right\}$. That this hypothesis on the $\left\{c_{j}\right\}$ is essential can be seen by considering the example with $c_{1}=1, c_{2}=-1, F_{1}(z)=\sin z$, and $F_{2}(z)=\cos z$. For these choices, easy calculations yield

$$
\left|\sum_{j=1}^{2} c_{j} n\left(r, 0, F_{j}\right)\right| \equiv 1
$$

and

$$
\left|\sum_{j=1}^{2} c_{j} r e^{i \theta} \frac{F_{j}^{\prime}\left(r e^{i \theta)}\right.}{F_{j}\left(r e^{i \theta}\right)}\right|=O\left(r e^{-2 r|\sin \theta|}\right), \quad r \rightarrow \infty,
$$

implying $m\left(U_{r}\right) \rightarrow 0$ as $r \rightarrow \infty$ for any choice of $\beta$ in $(0,1)$.

Secondly, we note in the case $L=1$ that Theorem 1 indeed provides a lower bound in terms of $n(r, 0, F)$ for $\left|r e^{i \theta} F^{\prime}\left(r e^{i \theta}\right) / F\left(r e^{i \theta}\right)\right|$. Theorem 1 with $L=1$ should be compared with earlier results of Hellerstein, Miles, and Rossi 
[9, Lemma 4], and of Fuchs [6, (17)]. Each of these results, after appropriate modifications, implies $m\left(U_{r}\right)>\delta>0$ as $r$ tends to $\infty$ through a sizeable set of values, but neither appears to give an estimate for $\delta$ comparable to (1.1). Perhaps more importantly, neither extends to linear combinations of logarithmic derivatives, a feature of Theorem 1 critical for our application in Section 3.

The elementary example $c_{1}=c_{2}=1$ with $F_{1}=\exp P$ and $F_{2}=\exp (-P)$ for any polynomial $P$ shows that while a lower bound for

$$
\left|\sum_{j=1}^{L} c_{j} r e^{i \theta} \frac{F_{j}^{\prime}\left(r e^{i \theta)}\right.}{F_{j}\left(r e^{i \theta}\right)}\right|
$$

is available in terms of the functionals $n\left(r, 0, F_{j}\right)$, no such bound is possible in terms of other measures of growth such as the characteristics $T\left(r, F_{j}\right)$ or their derivatives. Here again comparison with $[\mathbf{9}$, Lemma 4$]$ and $[6,(17)]$ is worthwhile, as each of these estimates for $\left|z F^{\prime}(z) / F(z)\right|$ is given in terms of growth functionals other than $n(r, 0, F)$.

Finally, Theorem 1 fails for entire functions of infinite order even in the case $L=1$. Such an example can be constructed by modifying MittagLeffler's function $[4$, p. 50]

$$
E_{\alpha}(z)=\sum_{n=0}^{\infty} \frac{z^{n}}{\Gamma(1+\alpha n)}, \quad 0<\alpha<2 .
$$

$E_{\alpha}(z)$ is an entire function of order $1 / \alpha$ for which, with $L=1$ and $\beta \in$ $(0,1)$ arbitrary, $U_{r} \subset(-(1 / 2+o(1)) \alpha \pi,(1 / 2+o(1)) \alpha \pi)$. By considering a sequence $\alpha_{k}$ tending to zero and by considering appropriate large sections of the Maclaurin series of $E_{\alpha_{k}}$, one can construct (by specifying the Maclaurin series) an entire function of infinite order for which there exists $E \subset[1, \infty)$ with upper logarithmic density 1 for which $U_{r}$ (with $L=1$ and $\beta \in(0,1)$ arbitrary) satisfies $m\left(U_{r}\right) \rightarrow 0$ as $r \rightarrow \infty, r \in E$.

In Section 3 we apply Theorem 1 to prove

Theorem 2. Consider the differential equation

$$
y^{(k)}+\sum_{j=0}^{k-2} A_{j} y^{(j)}=0, \quad k \geq 3,
$$

where $A_{j}(z)$ are entire, the order $\rho\left(A_{0}\right)$ of $A_{0}$ is not greater than $1 / 2$, and $\rho\left(A_{j}\right)<\rho\left(A_{0}\right)$ for $1 \leq j \leq k-2$. Then (1.2) cannot have two linearly independent solutions each with zero sequence having finite exponent of convergence.

Earlier Bank and Langley [2] obtained this result under the assumption that $\rho\left(A_{j}\right)<\rho\left(A_{0}\right)<1 / 2$ for $1 \leq j \leq k-2$. Our approach combines 
their method with Theorem 1 . It is known [1, p. 165] that Theorem 2 does not hold if $\rho\left(A_{0}\right)=1$. The question of whether Theorem 2 holds if $1 / 2<$ $\rho\left(A_{0}\right)<1$ remains open. Theorem 2 is already known in the case $k=2$. This was established in [1] for $\rho\left(A_{0}\right)<1 / 2$ and, independently, in [11] and [12] when $\rho\left(A_{0}\right)=1 / 2$.

In Section 4 we extend two earlier results of Langley concerning solutions of linear differential equations with entire coefficients where the dominant coefficient has order strictly less than $1 / 2$. Our extensions to the case where the dominant coefficient has order $1 / 2$ require some of the ideas involved in the proof of Theorem 2, but do not require Theorem 1. Because the arguments parallel those of Langley very closely, we provide only sketches of the proofs.

We assume throughout familiarity with the usual concepts and notation of value distribution theory.

\section{Proof of Theorem 1.}

We begin with a growth lemma establishing the existence of what may be regarded as sequences of modified Pólya peaks.

Lemma. Let $n(t)$ be a nondecreasing integer-valued function of order at most $\rho, 0 \leq \rho<\infty$, continuous from the right. Suppose $M>3$ and suppose $R_{0}>0$ is such that $n\left(R_{0}\right)>0$ and $n(t)=0$ for $0 \leq t<R_{0}$. Then there exists $E=E_{M} \subset\left[R_{0}, \infty\right)$ having lower logarithmic density at least $1-3 / M$ such that if $r \in E$, then

$$
n(t) \leq n(r)(t / r)^{2 M(\rho+1)}, \quad t \geq r
$$

and

$$
n(t) \geq n(r)(t / r)^{2 M(\rho+1)}, \quad R_{0} \leq t \leq r .
$$

Proof. We define

$$
A=\left\{r \geq R_{0}: \exists t=t_{r}>r \ni n(t) \geq n(r)(t / r)^{M(\rho+1)}\right\} .
$$

It is trivial that (2.1) holds for $r \in\left[R_{0}, \infty\right)-A$, and our first objective is to show that

$$
\overline{\log \operatorname{dens}} A<1 / M .
$$

Certainly (2.3) holds if $A$ is bounded, and we thus concern ourselves with the case that $A$ is unbounded. 
For each $r \in A$, let

$$
r^{*}=\sup \left\{t>r: n(t) \geq n(r)(t / r)^{M(\rho+1)}\right\} .
$$

We note that $r^{*}<\infty$ since $n(t)$ has order at most $\rho$. Since $n(t)$ is nondecreasing, we certainly have

$$
n\left(r^{*}\right) \geq n(r)\left(r^{*} / r\right)^{M(\rho+1)}
$$

for $r \in A$.

Let $r_{0}=\inf A$. Certainly $r_{0} \in A$ since $n(t)$ is continuous from the right and integer-valued with $n(t) / t^{M(p+1)}$ approaching 0 as $t$ approaches $\infty$. Letting

$$
r_{1}=\inf \left(A \cap\left(r_{0}^{*}, \infty\right)\right)
$$

we similarly note that $r_{1} \in A$. Trivially $r_{1} \geq r_{0}^{*}$. In fact $r_{1}>r_{0}^{*}$ in view of (2.4), (2.5), and the fact that $r_{1} \in A$. We define the sequence $r_{k}$ inductively by

$$
r_{k+1}=\inf \left(A \cap\left(r_{k}^{*}, \infty\right)\right), \quad k=0,1,2 \ldots,
$$

noting at each step that $A \cap\left(r_{k}^{*}, \infty\right) \neq \phi, r_{k} \in A$, and $r_{k+1}>r_{k}^{*}$.

Certainly $r_{k} \rightarrow \infty$ since $n\left(r_{k}^{*}\right)>n\left(r_{k}\right)$ and $n(t)$ is integer-valued. Thus

$$
A \subset \bigcup_{k=1}^{\infty}\left[r_{k}, r_{k}^{*}\right] \text {. }
$$

Since $r_{k} \in A$, we have by (2.5) for all $k$ that

$$
\log r_{k}^{*}-\log r_{k} \leq \frac{\log n\left(r_{k}^{*}\right)-\log n\left(r_{k}\right)}{M(\rho+1)}
$$

Combining these two observations and recalling that the intervals $\left[r_{k}, r_{k}^{*}\right]$ are disjoint, we have

$$
\begin{aligned}
\overline{\log \operatorname{dens}} A & \leq \varlimsup_{k \rightarrow \infty} \frac{\sum_{j=1}^{k} \log r_{j}^{*}-\log r_{j}}{\log r_{k}^{*}} \\
& \leq \frac{1}{M(\rho+1)} \varlimsup_{k \rightarrow \infty} \sum_{j=1}^{k} \frac{\log n\left(r_{j}^{*}\right)-\log n\left(r_{j}\right)}{\log r_{k}^{*}} \\
& \leq \frac{1}{M(\rho+1)} \varlimsup_{k \rightarrow \infty} \frac{\log n\left(r_{k}^{*}\right)-\log n\left(r_{1}\right)}{\log r_{k}^{*}}<\frac{1}{M}
\end{aligned}
$$


We now consider (2.2). Let

$$
B=\left\{r \geq R_{0}: \exists t=t_{r} \in\left[R_{0}, r\right) \ni n(t) \leq n(r)(t / r)^{2 M(\rho+1)}\right\} .
$$

It is elementary due to order considerations that $B$ is bounded if $A$ is bounded. Our goal is to show that

$$
\overline{\log \operatorname{dens}} B<2 / M,
$$

and thus we may assume that $A$ is unbounded.

Defining

$$
r_{j}^{* *}=\left(r_{j}^{*}\right)^{2} / r_{j}
$$

we first establish that

$$
B \subset \bigcup_{j=1}^{\infty}\left[r_{j}, r_{j}^{* *}\right]
$$

We suppose

$$
r \in B-\bigcup_{j=1}^{\infty}\left[r_{j}, r_{j}^{* *}\right]
$$

and seek a contradiction. Since $r \in B$, there exists $t=t_{r} \in\left[R_{0}, r\right)$ such that

$$
n(t) \leq n(r)(t / r)^{2 M(\rho+1)}<n(r)(t / r)^{M(\rho+1)} .
$$

Thus $t \in A$ and we may suppose $t \in\left[r_{j}, r_{j}^{*}\right]$. Consequently

$$
r>r_{j}^{* *}=\left(r_{j}^{*}\right)^{2} / r_{j} \geq t^{2} / r_{j}
$$

which implies

$$
t / r<\left(r_{j} / r\right)^{1 / 2}
$$

From (2.8) we conclude

$$
n\left(r_{j}\right) \leq n(t) \leq n(r)(t / r)^{2 M(\rho+1)} \leq n(r)\left(r_{j} / r\right)^{M(\rho+1)},
$$

implying $r \leq r_{j}^{*}$, which is a contradiction and thus establishes (2.7).

Since $\log \left(r_{j}^{* *} / r_{j}\right)=2 \log \left(r_{j}^{*} / r_{j}\right)$ and the upper logarithmic density of the union of the disjoint intervals $\left[r_{j}, r_{j}^{*}\right]$ is shown in (2.6) to be less than $1 / M$, we conclude from (2.7) that

$$
\overline{\log \operatorname{dens}} B \leq \overline{\log \operatorname{dens}} \bigcup_{j=1}^{\infty}\left[r_{j}, r_{j}^{* *}\right]<2 / M .
$$


We remark that the intervals $\left[r_{j}, r_{j}^{* *}\right]$ need not be disjoint.

Finally set

$$
E=\left[R_{0}, \infty\right)-(A \cup B) .
$$

Then the lower logarithmic density of $E$ is at least $1-3 / M$ and (2.1) and (2.2) hold for all $r \in E$.

Proof of Theorem 1. Let $n_{j}(t)=n\left(t, 0, F_{j}\right)$ and let

$$
n(t)=\sum_{j=1}^{L} n_{j}(t)
$$

Without loss of generality we may suppose $F_{j}(0) \neq 0$ for $1 \leq j \leq L$. (This can be verified easily be considering separately the cases where $n(t)$ is bounded and $n(t)$ is unbounded.) Trivially we may suppose $n(t) \not \equiv 0$.

For $1 \leq j \leq L$ such that $n_{j}(t) \equiv 0$, define $E(j)=[0, \infty)$ and $R_{0}(j)=0$. For all other $j, 1 \leq j \leq L$, let $R_{0}(j)$ be the modulus of the zero of $F_{j}$ of smallest modulus and apply the Lemma to $n_{j}(t)$ to obtain a set $E(j)$ of lower logarithmic density at least $1-3 / M$ on which $n_{j}(t)$ satisfies (2.1) and (2.2) with $R_{0}$ replaced by $R_{0}(j)$. We let

$$
R_{0}^{\prime}=\max _{j=1}^{L} R_{0}(j) \text { and } E^{\prime}=\bigcap_{j=1}^{L} E(j),
$$

and note that each $n_{j}(t)$ satisfies (2.1) and (2.2) on $E^{\prime}$ and that $E^{\prime}$ has lower logarithmic density at least $1-3 L / M$.

Let $q=[2 M(\rho+1)+2]$ and let $z_{\nu j}$ denote the zeros of $F_{j}$. We write

$$
F_{j}(z)=e^{P_{j}(z)} \prod_{\nu} E\left(\frac{z}{z_{\nu j}}, q\right)
$$

where $P_{j}$ is a polynomial of degree at most $q$. (The reader will note that this is not the usual Hadamard factorization.) We let

$$
E=\left(\left[2 R_{0}^{\prime}+1, \infty\right) \cap E^{\prime}\right)-\left\{\left|z_{\nu j}\right|: 1 \leq j \leq L, \nu \geq 1\right\} .
$$

For $r \in E$ we have

$$
r e^{i \theta} \frac{F_{j}^{\prime}\left(r e^{i \theta}\right)}{F_{j}\left(r e^{i \theta}\right)}=r e^{i \theta} P_{j}^{\prime}\left(r e^{i \theta}\right)+\sum_{m=-\infty}^{\infty} b_{m}\left(r, F_{j}\right) e^{i m \theta},
$$

where $b_{m}\left(r, F_{j}\right)=0$ for all $m$ if $n_{j}(t) \equiv 0$ and otherwise [13, p. 350]

$$
b_{m}\left(r, F_{j}\right)=-\sum_{\left|z_{\nu j}\right|>r}\left(r / z_{\nu j}\right)^{m}, \quad m>q
$$


and

$$
b_{m}\left(r, F_{j}\right)=\sum_{\left|z_{\nu j}\right| \leq r}\left(z_{\nu j} / r\right)^{-m}, \quad m<0 .
$$

For $m>q$ we have by (2.1) for $r \in E$ and for $1 \leq j \leq L$ (including those $j$ for which $\left.n_{j}(t) \equiv 0\right)$ that

$$
\begin{aligned}
\left|b_{m}\left(r, F_{j}\right)\right| & \leq \sum_{\left|z_{\nu j}\right|>r}\left(\frac{r}{\left|z_{\nu j}\right|}\right)^{m} \leq \int_{r}^{\infty}\left(\frac{r}{t}\right)^{m} d n_{j}(t) \\
& =-n_{j}(r)+m \int_{r}^{\infty}\left(\frac{r}{t}\right)^{m} \frac{n_{j}(t)}{t} d t \\
& \leq-n_{j}(r)+m n_{j}(r) \int_{r}^{\infty}\left(\frac{r}{t}\right)^{m}\left(\frac{t}{r}\right)^{2 M(\rho+1)} \frac{d t}{t} \\
& =n_{j}(r) \frac{2 M(\rho+1)}{m-2 M(\rho+1)} .
\end{aligned}
$$

Thus

$$
\sum_{m>q}\left|b_{m}\left(r, F_{j}\right)\right|^{2} \leq \frac{\pi^{2}}{6}\left(2 M(\rho+1) n_{j}(r)\right)^{2}, \quad 1 \leq j \leq L .
$$

Now consider $b_{m}\left(r, F_{j}\right)$ for $r \in E$ and $m<0$. From (2.2) we have for $1 \leq j \leq L$

$$
\begin{aligned}
\left|b_{m}\left(r, F_{j}\right)\right| & \leq \sum_{\left|z_{\nu j}\right| \leq r}\left(\frac{\left|z_{\nu j}\right|}{r}\right)^{-m}=\int_{R_{0}(j) / 2}^{r}\left(\frac{t}{r}\right)^{|m|} d n_{j}(t) \\
& =n_{j}(r)-|m| \int_{R_{0}(j)}^{r}\left(\frac{t}{r}\right)^{|m|} \frac{n_{j}(t)}{t} d t \\
& \leq n_{j}(r)-|m| n_{j}(r) \int_{R_{0}(j)}^{r}\left(\frac{t}{r}\right)^{|m|}\left(\frac{t}{r}\right)^{2 M(\rho+1)} \frac{d t}{t} \\
& =n_{j}(r)\left(\frac{2 M(\rho+1)}{|m|+2 M(\rho+1)}+\left(\frac{R_{0}(j)}{r}\right)^{|m|}\right) \\
& <n_{j}(r) \frac{4 M(\rho+1)}{|m|+2 M(\rho+1)},
\end{aligned}
$$

where in the last step we have used the fact that $M>3$ and used (2.9) to infer that $R_{0}(j) / r<\frac{1}{2}$. Thus for $r \in E$

$$
\sum_{m<0}\left|b_{m}\left(r, F_{j}\right)\right|^{2} \leq \frac{\pi^{2}}{6}\left(4 M(\rho+1) n_{j}(r)\right)^{2}, \quad 1 \leq j \leq L .
$$


For $r \in E$ we thus have for $1 \leq j \leq L$ that

$$
r e^{i \theta} \frac{F_{j}^{\prime}\left(r e^{i \theta}\right)}{F_{j}\left(r e^{i \theta}\right)}=\sum_{m=0}^{q} B_{m}\left(r, F_{j}\right) e^{i m \theta}+s_{j}(r, \theta)
$$

where

$$
\left\|s_{j}\right\|_{2} \leq 2 \pi M(\rho+1) n_{j}(r)
$$

by (2.11) and (2.12). Here if

$$
P_{j}(z)=\sum_{n=0}^{q} a_{n j} z^{n}
$$

then by $(2.10)$

$$
B_{m}\left(r, F_{j}\right)=b_{m}\left(r, F_{j}\right)+m a_{m j} r^{m}, \quad 0 \leq m \leq q .
$$

Let

$$
\tilde{U}_{r}=[0,2 \pi]-U_{r}
$$

and, for $0 \leq m \leq q$,

$$
d_{m}(r)=\sum_{j=1}^{L} c_{j} B_{m}\left(r, F_{j}\right)
$$

From (2.13) we thus obtain

$$
\sum_{j=1}^{L} c_{j} r e^{i \theta} \frac{F_{j}^{\prime}\left(r e^{i \theta}\right)}{F_{j}\left(r e^{i \theta}\right)}=\sum_{m=0}^{q} d_{m}(r) e^{i m \theta}+\sum_{j=1}^{L} c_{j} s_{j}(r, \theta)
$$

and note for $r \in E$ and $\theta \in \tilde{U}_{r}$ that

$$
|| \sum_{m=0}^{q} d_{m}(r) e^{i m \theta}|-| \sum_{j=1}^{L} c_{j} s_{j}(r, \theta)|| \leq \beta \sum_{j=1}^{L}\left|c_{j}\right| n_{j}(r) .
$$

We now set

$$
\left|\sum_{m=0}^{q} d_{m}(r) e^{i m \theta}\right|^{2}=\sum_{m=-q}^{q} h_{m}(r) e^{i m \theta}
$$

and note for $0 \leq m \leq q$ that $h_{-m}(r)=\overline{h_{m}(r)}$ and that

$$
\left|h_{m}(r)\right|=\left|\sum_{k=0}^{q-m} d_{m+k}(r) \overline{d_{k}(r)}\right| \leq \sum_{k=0}^{q}\left|d_{k}(r)\right|^{2}=h_{0}(r) .
$$


We have for $-q \leq m \leq q, m \neq 0$, that

$$
\int_{\tilde{U}_{r}} h_{m}(r) e^{i m \theta} d \theta=-\int_{U_{r}} h_{m}(r) e^{i m \theta} d \theta
$$

Thus by (2.17)

$$
\begin{aligned}
& \int_{\tilde{U}_{r}}\left|\sum_{m=0}^{q} d_{m}(r) e^{i m \theta}\right|^{2} d \theta \\
& =h_{0}(r) \int_{\tilde{U}_{r}} d \theta-\sum_{1 \leq|m| \leq q} \int_{U_{r}} h_{m}(r) e^{i m \theta} d \theta \\
& \geq h_{0}(r)\left(m\left(\tilde{U}_{r}\right)-2 q m\left(U_{r}\right)\right),
\end{aligned}
$$

where in the last line $m$ of course refers to Lebesgue measure.

For $r \in E$ for which (1.1) is not satisfied, we conclude from (2.18) that

$$
h_{0}(r)<\frac{1}{\pi} \int_{\tilde{U}_{r}}\left|\sum_{m=0}^{q} d_{m}(r) e^{i m \theta}\right|^{2} d \theta
$$

The combination of (2.14), (2.16), and (2.19) implies

$$
\begin{aligned}
\left(h_{0}(r)\right)^{\frac{1}{2}} & \leq \sqrt{2}\left(\frac{1}{2 \pi} \int_{\tilde{U}_{r}}\left|\sum_{m=0}^{q} d_{m}(r) e^{i m \theta}\right|^{2} d \theta\right)^{\frac{1}{2}} \\
& \leq \sqrt{2}\left(\left(\frac{1}{2 \pi} \int_{0}^{2 \pi}\left|\sum_{j=1}^{L} c_{j} s_{j}(r, \theta)\right|^{2} d \theta\right)^{\frac{1}{2}}+\beta \sum_{j=1}^{L}\left|c_{j}\right| n_{j}(r)\right) \\
& \leq \sqrt{2} \sum_{j=1}^{L}(2 \pi M(\rho+1)+\beta)\left|c_{j}\right| n_{j}(r) .
\end{aligned}
$$

From the definition of $U_{r}$ and the argument principle we have for $r \in E$ 
that

$$
\begin{aligned}
\left|\sum_{j=1}^{L} c_{j} n_{j}(r)\right| & =\left|\sum_{j=1}^{L} \frac{c_{j}}{2 \pi} \int_{0}^{2 \pi} r e^{i \theta} \frac{F_{j}^{\prime}\left(r e^{i \theta}\right)}{F_{j}\left(r e^{i \theta}\right)} d \theta\right| \\
& \leq \frac{1}{2 \pi} \int_{\tilde{U}_{r}}\left|\sum_{j=1}^{L} c_{j} r e^{i \theta} \frac{F_{j}^{\prime}\left(r e^{i \theta}\right)}{F_{j}\left(r e^{i \theta}\right)}\right| d \theta \\
& +\frac{1}{2 \pi} \int_{U_{r}}\left|\sum_{j=1}^{L} c_{j} r e^{i \theta} \frac{F_{j}^{\prime}\left(r e^{i \theta}\right)}{F_{j}\left(r e^{i \theta}\right)}\right| d \theta \\
& <\beta\left|\sum_{j=1}^{L} c_{j} n_{j}(r)\right|+\frac{1}{2 \pi} \int_{U_{r}}\left|\sum_{j=1}^{L} c_{j} r e^{i \theta} \frac{F_{j}^{\prime}\left(r e^{i \theta}\right)}{F_{j}\left(r e^{i \theta}\right)}\right| d \theta .
\end{aligned}
$$

Rearranging this inequality and appealing to (2.14), (2.15), (2.17), (2.20), and the Schwarz Inequality, we find for $r \in E$ that

$$
\begin{aligned}
& (1-\beta)\left|\sum_{j=1}^{L} c_{j} n_{j}(r)\right| \\
& <\left(\frac{m\left(U_{r}\right)}{2 \pi}\right)^{\frac{1}{2}}\left[\left(\sum_{k=0}^{q}\left|d_{k}(r)\right|^{2}\right)^{\frac{1}{2}}+\sum_{j=1}^{L} 2 \pi M(\rho+1)\left|c_{j}\right| n_{j}(r)\right] \\
& \leq\left(\frac{m\left(U_{r}\right)}{2 \pi}\right)^{\frac{1}{2}}\left[\sum_{j=1}^{L}(\sqrt{2} 2 \pi M(\rho+1)+\beta \sqrt{2}+2 \pi M(\rho+1))\left|c_{j}\right| n_{j}(r)\right] \\
& \leq 7\left(m\left(U_{r}\right)\right)^{\frac{1}{2}} \sum_{j=1}^{L} M(\rho+1)\left|c_{j}\right| n_{j}(r) .
\end{aligned}
$$

The hypothesis on the arguments of the $\left\{c_{j}\right\}$ implies

$$
\left|\sum_{j=1}^{L} c_{j} n_{j}(r)\right| \geq(\cos \gamma) \sum_{j=1}^{L}\left|c_{j}\right| n_{j}(r) .
$$

Combining the last two inequalities yields (1.1) for $r \in E$, proving Theorem 1.

\section{An Application.}

Our proof of Theorem 2 is a modification of the proof of the corresponding result in [2] in the case that $\rho\left(A_{0}\right)<1 / 2$. The additional ingredients in our proof are Theorem 1 and results concerning the behavior of entire functions 
of order $1 / 2$ previously applied in [8] and [9] to the growth of solutions of certain linear differential equations. We concern ourselves only with the case $\rho\left(A_{0}\right)=1 / 2$.

We begin by recalling two key aspects of the argument in. [2]. The first is an upper bound for the modulus of the logarithmic derivative of a meromorphic function of finite order outside of a small exceptional set. If $G$ is meromorphic of finite order in the plane, $k$ is a positive integer, and $d>0$, then there exists a sequence of disks

$$
D_{n}=\left\{z:\left|z-z_{n}\right|<\left|z_{n}\right|^{-d}\right\}
$$

and a constant $M>0$ such that

$$
\left|G^{(j)}(z) / G(z)\right|<|z|^{M}
$$

for all $z$ of large modulus in $C-\bigcup_{n=1}^{\infty} D_{n}$ and for $1 \leq j \leq k$. The sequence $z_{n}$ and the constant $d>0$ can be chosen so that the sum of the radii of the disks is finite. When this sum is finite, we shall call the union $D$ of the sequence of disks $D_{n}$ the $R$-set associated with the sequence $z_{n}$ and the constant $d>0$. The centers $z_{n}$ of the disks $D-n$ comprising the $R$-set may in fact be chosen to be the poles of $G$ and the zeros of $G^{(j)}, 0 \leq j \leq k-1$. The estimate (3.1) follows from straightforward calculations based on the differentiated Poisson-Jensen formula [7, p. 22].

Let $C_{r}:=\{z:|z|=r\}$, and, for a given $R$-set $D$, let

$$
L_{r}:=\left\{\theta \in[0,2 \pi): r e^{i \theta} \in D\right\} .
$$

Let $G$ be meromorphic of order $\rho$ and let $\epsilon>0$. If $D$ is the $R$-set associated with the constant $d>\rho$ and with the poles of $G$ and the zeros of any finite number of its derivatives, then using order considerations and elementary geometry it is easy to verify that

$$
m\left(L_{r}\right)=o\left(r^{\rho+\epsilon-d-1}\right) .
$$

The second element of the argument in [2] we wish to recall follows from the classical $\cos \pi \rho$ theorem combined with results of Barry [3]. If $\tau<$ $\rho\left(A_{0}\right)<1 / 2$ and $D$ denotes an $R$-set associated with the zero and pole sets of any finite collection of meromorphic functions of finite order, then there exists $r_{n} \rightarrow \infty$ for which

$$
D \cap C_{r_{n}}=\phi
$$

and

$$
\log \left|A_{0}\left(r_{n} e^{i \theta}\right)\right|>r_{n}^{\tau}, \quad 0 \leq \theta \leq 2 \pi
$$


Hellerstein, Miles, and Rossi observed, based on a result of Drasin and Shea [5, Theorem 8.1], that a variant of (3.3) holds if $\rho\left(A_{0}\right)=1 / 2$. Specifically, in [8, Section 2] it is shown that if $\rho\left(A_{0}\right)=1 / 2$ and $\tau<1 / 2$ then one of two possibilities must occur. The first (which throughout the remainder of the paper we shall call Case I) is that there exists $r_{n} \rightarrow \infty$ for which (3.3) holds. The second possibility (henceforth designated Case II) is that there exists $E \subset[1, \infty)$ of logarithmic density one such that for $r \in E$

$$
\left\{\theta \in[0,2 \pi): \log \left|A_{0}\left(r e^{i \theta}\right)\right|<r^{r}\right\} \subset H_{r},
$$

where $H_{r}$ is some single interval (modulo $2 \pi$ ) satisfying $m\left(H_{r}\right) \rightarrow 0$ as $r \rightarrow \infty, r \in E$.

For later use we now make an elementary estimate concerning the characteristic of an entire function $G$. Suppose $J_{r} \subset[0,2 \pi)$ and $\tilde{J}_{r}=[0,2 \pi)-J_{r}$. Then we have

$$
\begin{aligned}
T(r, G) & =\frac{1}{2 \pi} \int_{J_{r}} \log ^{+}\left|G\left(r e^{i \theta}\right)\right| d \theta+\frac{1}{2 \pi} \int_{\tilde{J}_{r}} \log ^{+}\left|G\left(r e^{i \theta}\right)\right| d \theta \\
& \leq \frac{m\left(J_{r}\right)}{2 \pi} \log ^{+} M(r, G)+\frac{1}{2 \pi} \int_{\tilde{J}_{r}} \log ^{+}\left|G\left(r e^{i \theta}\right)\right| d \theta \\
& \leq \frac{3 m\left(J_{r}\right)}{2 \pi} T(2 r, G)+\frac{1}{2 \pi} \int_{\tilde{J}_{r}} \log ^{+}\left|G\left(r e^{i \theta}\right)\right| d \theta
\end{aligned}
$$

If in addition there exists $M>0$ such that $\left|G\left(r e^{i \theta}\right)\right|<r^{M}$ for $\theta \in \tilde{J}_{r}$, we may further conclude

$$
T(r, G)<\frac{3 m\left(J_{r}\right)}{2 \pi} T(2 r, G)+O(\log r) .
$$

Following [2], we now suppose that

$$
f_{1}=V e^{g} \quad \text { and } \quad f_{2}=W e^{h}
$$

are linearly independent solutions of (1.2) whose zero sequences have finite exponent of convergence. By standard arguments [2, Section 2] we may suppose $V, W, g$, and $h$ all have finite order. In [2] it is shown that to obtain the desired contradiction, it is sufficient to show that $f_{1} / f_{2}$ has finite order.

We let $d>0$ be such that $d / 2$ is greater than the maximum $\rho$ of the orders of $V, W, g$, and $h$. Let $D$ be the $R$-set associated with $d$ and the sequence $z_{n}$ formed from the zeros of $V^{(j)}, W^{(j)}, g^{(j)}$, and $h^{(j)}$ for $0 \leq j \leq k-1$. From (3.1) we conclude there exists $M>0$ such that

$$
\left|\frac{W^{\left(m_{1}\right)}(z)}{W(z)}\right|+\left|\frac{V^{\left(m_{2}\right)}(z)}{V(z)}\right|+\left|\frac{g^{\left(m_{3}\right)}(z)}{g^{\prime}(z)}\right|+\left|\frac{h^{\left(m_{4}\right)}(z)}{h^{\prime}(z)}\right|=O\left(|z|^{M}\right)
$$


for $z \notin D$ and all choices of $m_{j}, 1 \leq m_{j} \leq k$. We note for this $R$-set and this choice of $\rho$ and $d$ that (3.2) holds with any choice of $\epsilon>0$. Using the principal hypothesis of Theorem 2, we now choose $\epsilon>0$ to satisfy

$$
\max _{j=1}^{k-2} \rho\left(A_{j}\right)<1 / 2-4 \epsilon \text {. }
$$

Following the argument in [2], we insert $y=f_{1}$ into (1.2) and collect terms to obtain

$$
\left(g^{\prime}\right)^{k}+\left(k \frac{V^{\prime}}{V}+\frac{k(k-1)}{2} \frac{g^{\prime \prime}}{g^{\prime}}\right)\left(g^{\prime}\right)^{k-1}+\sum_{j=0}^{k-2} B_{j}\left(g^{\prime}\right)^{j}+A_{0}=0
$$

where each $B_{j}$ is a polynomial in $A_{1}, A_{2}, \ldots, A_{k-2}$ and the logarithmic derivatives $V^{(m)} / V$ and $g^{(m)} / g^{\prime}$ for $1 \leq m \leq k-1$. Similarly, the insertion of $y=f_{2}$ into (1.2) yields

$$
\left(h^{\prime}\right)^{k}+\left(k \frac{W^{\prime}}{W}+\frac{k(k-1)}{2} \frac{h^{\prime \prime}}{h^{\prime}}\right)\left(h^{\prime}\right)^{k-1}+\sum_{j=0}^{k-2} \tilde{B}_{j}\left(h^{\prime}\right)^{j}+A_{0}=0
$$

where the $\tilde{B}_{j}$ are polynomials in $A_{1}, A_{2}, \ldots, A_{k-2}, W^{(m)} / W$, and $h^{(m)} / h^{\prime}$ for $1 \leq m \leq k-1$.

We now consider the two types of behavior which $A_{0}$ may exhibit (Case I or Case II). In each case we wish to establish three properties of $g$ and $h$. These are that (i) the lower order of $h^{\prime}$ is at most $1 / 2$, (ii) there exists a complex number $a,|a|=1$, such that $g-a h$ is a polynomial, and (iii) there exists $r_{\dot{n}} \rightarrow \infty$ such that $g^{\prime}$ dominates all coefficients of $\left(g^{\prime}\right)^{j}, 0 \leq j \leq k-1$, in (3.7) and $h^{\prime}$ dominates all coefficients of $\left(h^{\prime}\right)^{j}, 0 \leq j \leq k-1$, in (3.8) on all of $C_{r_{n}}$ in Case I and on most of $C_{r_{n}}$ in Case II.

We first consider Case I, i.e. we suppose that there exists $r_{n} \rightarrow \infty$ such that (3.3) holds with $\tau=1 / 2-\epsilon$. It is useful to have control of the quantity on the left side of (3.6) on all of $C_{r_{n}}$, and to this end we appeal to Lemma 6 of [9] to presume with no loss in generality that

$$
\left|\frac{W^{\left(m_{1}\right)}(z)}{W(z)}\right|+\left|\frac{V^{\left(m_{2}\right)}(z)}{V(z)}\right|+\left|\frac{g^{\left(m_{3}\right)}(z)}{g^{\prime}(z)}\right|+\left|\frac{h^{\left(m_{4}\right)}(z)}{h^{\prime}(z)}\right|=O\left(\exp |z|^{\epsilon}\right)
$$

for all $z \in C_{r_{n}}$ and all choices of $m_{j}, 1 \leq m_{j} \leq k$.

By (3.9) and our choie of $\epsilon$, we have

$$
\left|B_{j}(z)\right|+\left|\tilde{B}_{j}(z)\right|=o\left(\exp \left(|z|^{\frac{1}{2}-3 \epsilon}\right)\right), \quad z \in C_{r_{n}} .
$$

From (3.3), (3.7), (3.9), and (3.10) it is immediate that

$$
\left|g^{\prime}(z)\right|>\exp \left(|z|^{\frac{1}{2}-2 \epsilon}\right), \quad z \in C_{r_{n}}, n>n_{0} .
$$


Similarly (3.3), (3.8), (3.9), and (3.10) imply

$$
\left|h^{\prime}(z)\right|>\exp \left(|z|^{\frac{1}{2}-2 \epsilon}\right), \quad z \in C_{r_{n}}, n>n_{0} .
$$

From (3.9), (3.10), (3.11), and (3.12) we have for all $z \in C_{r_{n}}$ that

$$
\begin{aligned}
\left|\frac{V^{\prime}(z)}{V(z)}\right|+\left|\frac{g^{\prime \prime}(z)}{g^{\prime}(z)}\right|+\left|\frac{W^{\prime}(z)}{W(z)}\right|+ & \left|\frac{h^{\prime \prime}(z)}{h^{\prime}(z)}\right|+\left|B_{j}(z)\right|+\left|\tilde{B}_{j}(z)\right| \\
& =o\left(\exp \left(|z|^{-\epsilon}\right)\right) \min _{z \in C_{r_{n}}}\left(\left|h^{\prime}(z),\right| g^{\prime}(z) \mid\right) .
\end{aligned}
$$

In view of (3.13), division of (3.7) by $\left(g^{\prime}\right)^{k}$ yields

$$
\frac{A_{0}(z)}{\left(g^{\prime}(z)\right)^{k}}=-1+o(1), \quad z \in C_{r_{n}}
$$

Thus there exists an analytic $k$ th root $A_{0}(z)^{1 / k}$ of $A_{0}(z)$ defined on $C_{r_{n}}^{\prime}=$ $C_{r_{n}}-\left\{r_{n}\right\}$ and $a_{n}$ with $a_{n}^{k}=-1$ such that

$$
\frac{A_{0}(z)^{1 / k}}{g^{\prime}(z)}=a_{n}+o(1), \quad z \in C_{r_{n}} \text {. }
$$

We furthe note from (3.6) and (3.13) that for $z \in C_{r_{n}}-D$, (3.7) takes the form

$$
\frac{A_{0}(z)}{\left(g^{\prime}(z)\right)^{k}}=-1+O\left(\frac{|z|^{M}}{\left|g^{\prime}(z)\right|}\right) \text {. }
$$

We conclude

$$
\frac{A_{0}(z)^{1 / k}}{g^{\prime}(z)}=a_{n}+O\left(\frac{|z|^{M}}{\left|g^{\prime}(z)\right|}\right), \quad z \in C_{r_{n}}^{\prime}-D
$$

which we rearrage to obtain

$$
\left|g^{\prime}(z)-a_{n}^{-1} A_{0}(z)^{1 / k}\right|=O\left(|z|^{M}\right), \quad z \in C_{r_{n}}^{\prime}-D
$$

A similar analysis leads to the existence of $b_{n}$ with $b_{n}^{k}=-1$ such that

$$
\left|h^{\prime}(z)-b_{n}^{-1} A_{0}(z)^{1 / k}\right|=O\left(|z|^{M}\right), \quad z \in C_{r_{n}}-D .
$$

By passing to a subsequence we conclude there exists $a$ with $a^{k}=1$ such that

$$
\left|g^{\prime}(z)-a h^{\prime}(z)\right|=O\left(|z|^{M}\right)
$$


for $z \in C_{r_{n}}^{\prime}-D$. We apply (3.5) with $G=g^{\prime}-a h^{\prime}$ and $J_{r_{n}}=L_{r_{n}}$ to conclude from (3.2) and the definitions of $\rho$ and $d$ that

$$
\begin{aligned}
T\left(r_{n}, G\right) & <o\left(r_{n}^{\rho+\epsilon-d-1}\right)\left(2 r_{n}\right)^{\rho+\epsilon}+O\left(\log r_{n}\right) \\
& =o(1)+O\left(\log r_{n}\right)
\end{aligned}
$$

implying $G=g^{\prime}-a h^{\prime}$ is a polynomial.

We apply (3.4) to conclude that $h^{\prime}$ has lower order at most $1 / 2$. Indeed setting $G=h^{\prime}$, we have

$$
\begin{aligned}
T\left(r_{n}, h^{\prime}\right) & <\frac{3 m\left(L_{r_{n}}\right)}{2 \pi} T\left(2 r_{n}, h^{\prime}\right)+\frac{1}{2 \pi} \int_{\tilde{L}_{r_{n}}} \log ^{+}\left|h^{\prime}\left(r_{n} e^{i \theta}\right)\right| d \theta \\
& =o\left(r_{n}^{\rho+\epsilon-d-1}\right)\left(2 r_{n}\right)^{\rho+\epsilon}+o\left(r_{n}^{\frac{1}{2}+\epsilon}\right) \\
& =o\left(r_{n}^{\frac{1}{2}+\epsilon}\right),
\end{aligned}
$$

where we have used (3.2) and (3.15).

We next consider Case II. Since $E \subset[1, \infty)$ has logarithmic density one and $\left\{r: C_{r} \cap D \neq \emptyset\right\}$ has finite measure, with no loss of generality we may assume for all $r \in E$ that $C_{r} \cap D=\emptyset$. Recalling that $H_{r}$ is an interval modulo $2 \pi$, we let $A_{0}(z)^{1 / k}$ be an analytic $k$-th root of $A_{0}(z)$ for $z=r e^{i \theta}$, $r \in E$, and $\theta \notin H_{r}$. Our arguments from Case I apply to yield

$$
\left|B_{j}(z)\right|+\left|\tilde{B}_{j}(z)\right|=o\left(\exp \left(|z|^{\frac{1}{2}-3 \epsilon}\right)\right), \quad|z|=r, r \in E,
$$

and, for large $r$,

$$
\left|g^{\prime}(z)\right|>\exp \left(|z|^{\frac{1}{2}-2 \epsilon}\right), \quad z=r e^{i \theta}, r \in E, \theta \notin H_{r},
$$

and

$$
\left|h^{\prime}(z)\right|>\exp \left(|z|^{\frac{1}{2}-2 \epsilon}\right), \quad z=e^{i \theta}, r \in E, \theta \notin H_{r} .
$$

From (3.6), (3.16), (3.17), and (3.18) we conclude as before for $r \in E$ and $\theta \notin H_{r}$ that

$$
\begin{aligned}
\left|\frac{V^{\prime}\left(r e^{i \theta}\right)}{V\left(r e^{i \theta}\right)}\right|+\left|\frac{g^{\prime \prime}\left(r e^{i \theta}\right)}{g^{\prime}\left(r e^{i \theta}\right)}\right|+\left|\frac{W^{\prime}\left(r e^{i \theta}\right)}{W\left(r e^{i \theta}\right)}\right| & +\left|\frac{h^{\prime \prime}\left(r e^{i \theta}\right)}{h^{\prime}\left(r e^{i \theta}\right)}\right|+\left|B_{j}\left(r e^{i \theta}\right)\right|+\left|\tilde{B}_{j}\left(r e^{i \theta}\right)\right| \\
& =o\left(\exp \left(r^{-\epsilon}\right)\right) \min _{\theta \notin H_{r}}\left(\left|h^{\prime}\left(r e^{i \theta}\right)\right|,\left|g^{\prime}\left(r e^{i \theta}\right)\right|\right) .
\end{aligned}
$$

From (3.19) we deduce by reasoning similar to that in Case I that (3.14) and (3.15) hold for $z=r e^{i \theta}, r \in E$, and $\theta \notin H_{r}$ with $a_{n}$ and $b_{n}$ replaced by 
$a_{r}$ and $b_{r}$ satisfying $a_{r}^{k}=b_{r}^{k}=-1$. It follows that there exists $E_{1} \subset E$ with upper logarithmic density at least $1 / k$ and there exists $a$ with $a^{k}=1$ such that for $r \in E_{1}$

$$
\left|g^{\prime}(z)-a h^{\prime}(z)\right|=O\left(|z|^{M}\right), \quad z=r e^{i \theta}, \theta \notin H_{r} .
$$

An application of (3.5) with $G=g^{\prime}-a h^{\prime}$ and $J_{r}=H_{r}$ yields that if $G$ is not a polynomial then

$$
\lim _{\substack{r \rightarrow \infty \\ r \in E_{1}}} \frac{T(2 r, G)}{T(r, G)}=\infty
$$

implying that $G$ has infinite order since $E_{1}$ has positive logarithmic density. We conclude that $G$ is in fact a polynomial.

We now apply the ideas involved in (3.4) with $G=h^{\prime}$ and $J_{r}=H_{r}$ to deduce that the lower order of $h^{\prime}$ is at most 1/2. First suppose that there exists $\tilde{E} \subset E$ having positive upper logarithmic density for which

$$
\varliminf_{r \in \mathbb{E}} \underset{\substack{r \rightarrow \infty \\ H_{r}}}{ } \log ^{+}\left|h^{\prime}\left(r e^{i \theta}\right)\right| d \theta / T\left(r, h^{\prime}\right)>0 .
$$

Then the reasoning used to obtain (3.4) yields

$$
\lim _{\substack{r \rightarrow \infty \\ r \in \tilde{E}}} \frac{T\left(2 r, h^{\prime}\right)}{T\left(r, h^{\prime}\right)}=\infty,
$$

implying the order of $h^{\prime}$ is infinite. We conclude that there exists a set $E_{2} \subset E$ having logarithmic density one such that

$$
\int_{H_{r}} \log ^{+}\left|h^{\prime}\left(r e^{i \theta}\right)\right| d \theta=o\left(T\left(r, h^{\prime}\right)\right)
$$

for $r \in E_{2}$. From (3.4) and (3.15) (which in Case II holds for $z=r e^{i \theta}, r \in E$, and $\theta \notin H_{r}$ with $b_{n}$ replaced by $b_{r}$ ), we conclude that the lower order of $h^{\prime}$ is at most $1 / 2$.

This establishes our goal of showing in both Case I and Case II that $g^{\prime}-a h^{\prime}$ is a polynomial, that the lower order of $h^{\prime}$ is at most $1 / 2$, and that (3.13) holds in Case I for $z \in C_{r_{n}}$ and (3.19) holds in Case II for $r \in E$ and $\theta \notin H_{r}$.

Our argument now follows that of Bank and Langley very closely. We first show that without loss of generality we may presume in fact $g=a h$. Indeed note that $\tilde{V}=V e^{P}$ has finite order for any polynomial $P=g-a h$. Next observe in Case I that (3.9) holds with $V$ replaced by $\tilde{V}$ and thus as before that (3.13) holds for $z \in C_{r_{n}}$ In Case II our previous arguments apply to show that (3.19) holds for $z=r e^{i \theta}, r \in E_{3}$, and $\theta \notin H_{r}$ for a set $E_{3}$ of logarithmic density one. 
Having justified that (3.13) and (3.19) hold under the presumption that $g=a h$, we next show that $a=1$. This is sufficient to prove Theorem 2 , for $a=1$ implies $f_{1} / f_{2}=\tilde{V} / W$ has finite order, which as observed earlier is a contradiction. Clearly we need concern ourselves only with the situation in which $h$ is transcendental.

We substitute $f_{1}=\tilde{V} e^{a h}$ and $f_{2}=W e^{h}$ into (1.2) to conclude from (3.7), (3.8), (3.13), and (3.19) that

$$
\begin{aligned}
& \left(a h^{\prime}\right)^{k}+k\left(\frac{\tilde{V}^{\prime}}{\tilde{V}}\right)\left(a h^{\prime}\right)^{k-1}+\frac{k(k-1)}{2}\left(a h^{\prime}\right)^{k-2}\left(a h^{\prime \prime}\right) \\
& =\left(h^{\prime}\right)^{k}+k\left(\frac{W^{\prime}}{W}\right)\left(h^{\prime}\right)^{k-1}+\frac{k(k-1)}{2}\left(h^{\prime}\right)^{k-2} h^{\prime \prime}+O(|z|-2)\left(h^{\prime}\right)^{k-1}
\end{aligned}
$$

for $z \in C_{r_{n}}$ in Case I and $z=r e^{i \theta}, r \in E_{3}$, and $\theta \notin H_{r}$ in Case II. Since $a^{k}=1,(3.21)$ may be rearranged to obtain

$$
2 k \frac{\tilde{V}^{\prime}}{\tilde{V}}+k(k-1) \frac{h^{\prime \prime}}{h^{\prime}}-a\left(2 k \frac{W^{\prime}}{W}+k(k-1) \frac{h^{\prime \prime}}{h^{\prime}}\right)=O\left(|z|^{-2}\right) .
$$

We now let $F_{1}=\tilde{V}^{2 k}\left(h^{\prime}\right)^{k(k-1)}$ and $F_{2}=W^{2 k}\left(h^{\prime}\right)^{k(k-1)}$. We note that both $F_{1}$ and $F_{2}$ have zeros since the lower order of the transcendental function $h^{\prime}$ is at most $1 / 2$. In Case I we multiply both sides of (3.22) by $r_{n} e^{i \theta}$ and observe that the integral of the left side over $[0,2 \pi]$ cannot be $o(1)$ unless $a=1$ since $F_{1}$ and $F_{2}$ each have zeros. In Case II we recall that (3.22) is valid for all $r$ in a set of logarithmic density one. This fact, in combination with Theorem 1 applied to $F_{1}$ and $F_{2}$ with $c_{1}=1$ and $c_{2}=-a$, implies $a=1$. Thus $a=1$ in both cases, which as observed above is sufficient to prove the theorem.

\section{Concluding Remarks.}

In this section we indicate how our analysis of the two possible types of behavior of an entire function of order 1/2 (Case I and Case II) can be used to extend two previous results of Langley. We obtain the following theorems.

Theorem 3. For $k>3$, consider

$$
y^{(k)}+\sum_{j=0}^{k-2} A_{j} y^{(j)}=0,
$$

where $A_{j}$ is entire and there exists $s \in\{1,2, \ldots, k-2\}$ such that $\rho\left(A_{s}\right)=1 / 2$ and $\rho\left(A_{j}\right)<1 / 2$ for $0 \leq j \leq k-2, j \neq s$. Then (4.1) cannot have $k$ 
linearly independent solutions each of whose zero set has finite exponent of convergence.

Theorem 4. For $k>2$, consider

$$
y^{(k)}+A y^{(k-1)}+\sum_{j=0}^{k-2} B_{j} y^{(j)}=0
$$

where $\rho(A)=1 / 2$ and $\rho\left(B_{j}\right)<1 / 2$ for $0 \leq j \leq k-2$. Then the zero sequence of any transcendental solution of (4.2) has infinite exponent of convergence.

Earlier Langley [10] obtained each of these results under the assumption that the dominant coefficient has order strictly less than $1 / 2$.

We now sketch the minor modifications of Langley's arguments required to prove Theorem 3 and Theorem 4 . We designate the dominant coefficient in each theorem by $A$. A careful analysis of entire functions $A$ of order $1 / 2$ (see the discussion [9, p. 355]) shows that if Case II does not hold for $A$, then if $\tau<1 / 2$ there in fact exists $E^{\prime} \subset[1, \infty)$ of infinite measure such that

$$
\log \left|A\left(r e^{i \theta}\right)\right|>r^{\tau}
$$

for $r \in E^{\prime}$ and $0 \leq \theta \leq 2 \pi$. Thus if Case II does not hold for $A$ and $D$ is any $R$-set, there exists $r_{n} \rightarrow \infty, r_{n} \in E^{\prime}$, and $C_{r_{n}} \cap D=\phi$. Langley's arguments apply verbatim in this context for both Theorem 3 and Theorem 4 .

If Case II holds for $A$, then for any $R$-set $D$ and any $\tau<1 / 2$, there exists $E^{\prime} \subset[1, \infty)$ having logarithmic density one and such that for $r \in E^{\prime}$ we have $C_{r} \cap D=\phi$ and

$$
\log \left|A\left(r e^{i \theta}\right)\right|>r^{\tau}, \quad \theta \notin H_{r},
$$

where $m\left(H_{r}\right) \rightarrow 0$ and $H_{r}$ is an interval. It is routine to verify that Langley's estimates, which apply in his context on circles for which (3.3) holds with $A_{0}$ replaced by $A$, hold in our context on $C_{r}-\left\{r e^{i \theta}: \theta \notin H_{r}\right\}$ for $r \in E^{\prime}$. The only estimate needing additional comment is (6.9) in [10] in the proof of our Theorem 4, for which we now have

$$
\left|h^{\prime}(z)+A(z)\right|=O\left(|z|^{M}\right)
$$

for $z=r e^{i \theta}, r \in E^{\prime}$, and $\theta \notin H_{r}$. We apply (3.5) with $G=h^{\prime}+A$ to conclude by reasoning identical to that in (3.20) that $G$ is a polynomial.

Both Theorem 3 and Theorem 4 are proved by contradiction. A collection of linearly independent entire solutions (consisting of one member in the case of Theorem 4 and of $k$ members in the case of Theorem 3) having zero 
sequences with finite exponent of convergence is presumed to exist. For both Theorem 3 and Theorem 4 Langley's arguments produce an entire function $F$ (known to have zeros by construction) satisfying for $r \in E^{\prime}$

$$
\left|F^{\prime}\left(r e^{i \theta}\right) / F\left(r e^{i \theta}\right)\right|=o\left(r^{-2}\right), \quad \theta \notin H_{r} .
$$

This is impossible by Lemma 4 of [9] as well as by our Theorem 1, completing the proof of both theorems.

\section{References}

[1] S. Bank and I. Laine, On the oscillation theory of $f^{\prime \prime}+A f=0$ where $A$ is entire, Trans. Amer. Math. Soc., 273 (1982), 351-363.

[2] S. Bank and J. Langley, Oscillation theory of higher order linear differential equations with entire coefficients, Complex Variables, 16 (1991), 163-175.

[3] P. Barry, Some theorems related to the $\cos \pi \rho$ theorem, Proc. London Math. Soc. (3), 21 (1970), 344-360.

[4] M. Cartwright, Integral Functions, Cambridge University Press, 1956.

[5] D. Drasin and D. Shea, Convolution inequalities, regular variation and exceptional sets, J. Analyse Math., 29 (1976), 232-293.

[6] W. Fuchs, Proof of a conjecture of G. Polya concerning gap series, Ill. J. Math., 7 (1963), 661-667.

[7] W. Hayman, Meromorphic Functions, Oxford at the Clarendon Press, 1964.

[8] S. Hellerstein, J. Miles, and J. Rossi, On the growth of solutions of $f^{\prime \prime}+g f^{\prime}+h f=0$, Trans. Amer. Math. Soc., 324 (1991), 693-706.

[9] S. Hellerstein, J. Miles, and J. Rossi, On the growth of solutions of certain linear differential equations, Ann. Acad. Sci. Fenn., 17 (1992), 343-365.

[10] J. Langley, Some oscillation theorems for higher order linear differential equations with entire coefficients of small growth, Results in Math., 20 (1991), 517-529.

[11] J. Rossi, Second order differential equations with transcendental coefficients, Proc. Amer. Math. Soc., 97 (1986), 61-66.

[12] L.-C. Shen, Solution to a problem of $S$. Bank regarding the exponent of convergence of the solutions of a differential equation $f^{\prime \prime}+A f=0$, Kexue Tongbao, 30 (1985), 1581-1585.

[13] D. Townsend, Comparisons between $T(r, f)$ and the total variation of $\arg f\left(r e^{i \theta}\right)$ and $\log \left|f\left(r e^{i \theta}\right)\right|$, J. Math. Anal. and Appl., 128 (1987), 347-361.

Received August 4, 1993 and revised November 23, 1993.

UNIVERSITY OF ILLINOIS

URBANA, ILLINOIS 61801

E-mail address: joe@math.uiuc.edu

AND

Virginia Polytechnic Institute and State University

BLACKSBURG, VIRGINIA 24061

E-mail address: rossi@math.vt.edu 



\section{PACIFIC JOURNAL OF MATHEMATICS}

Volume $174 \quad$ No. $1 \quad$ May 1996

A distance formula for algebras on the disk

CHRISTOPHER J. BISHOP

Rigidity of isotropic maps

FERNANDO CUKIERMAN

The Schwartz space of a general semisimple Lie group. V. Schwartz

class wave packets

REBECCA A. HERB

Rational polynomials with a $\mathbf{C}^{*}$-fiber

SHULIM KALIMAN

Linear combinations of logarithmic derivatives of entire functions with 195 applications to differential equations

Joseph B. Miles and JoHn Rossi

Factorization problems in the invertible group of a homogeneous $C^{*}$-algebra

N. Christopher PHiLlips

Higher order estimates in complex interpolation theory

RICHARD ROCHBERG

Braid commutators and Vassiliev invariants

TED STANFORD

On the Cauchy problem for a singular parabolic equation 